LEMMA 6. If $\Sigma$ is recursive at $x$, then $S$ is recursive at $x$.

Proof. Let $U$ be an open neighborhood of $x$. By Lemma 4 there exists a compact set $M$ in $T$ such that $x M \subset U$ and $\Sigma \subset S M^{-1}$. Let $V$ be a neighborhood of $x$ for which $V M \subset U$. There exists an admissible set $A$ such that $A \subset \Sigma$ and $x A \subset V$. Hence $x A M \subset U$. Define $B=S \cap A M$. Since $A \subset B M^{-1}, B$ is an admissible set. Also $B \subset S$ and $x B \subset U$. The proof is completed.

The following theorem is an immediate consequence of Lemmas 5 and 6.

THEOREM. If $T$ is recursive at $x$, then $S$ is recursive at $x$.

An interpretation of admissibility arises if we define an admissible subset of $T$ to be a relatively dense subset of $T$. The term "recursive" is then replaced by "almost periodic." For other applications, see the paper cited above.

University of PenNSYLVAaria

\title{
FIXED POINT THEOREMS FOR INTERIOR TRANSFORMATIONS
}

\section{O. H. HAMILTON}

If $M$ is a bounded continuum in a Euclidean plane $E$ which does not separate $E$ and $T$ is an interior continuous transformation of $M$ onto a subset of $E$ which contains $M$, does $T$ leave a point of $M$ invariant? It is the purpose of this paper to answer this question in the affirmative for certain types of locally connected continua.

Using a notation introduced by Eilenberg $[2, \text { p. 168 }]^{1}$ a continuum $M$ will be said to have property (b) provided every continuous transformation of $M$ into the unit circle $S$ in the Cartesian plane, with center at $o$, is homotopic to a constant mapping, that is, a transformation which transforms each point of $M$ into a single point of $S$. If $T$ is a continuous transformation of a subset $A$ of the plane $E$ into a subset $B$ of $E$, then for each point $x$ of $A$ let $T^{\prime}(x)$ be the point $y$ of $S$ such that the directed line segment $o y$ is parallel in direction and sense to the directed line segment $x, T(x)$. Then $T^{\prime}$ will be referred to as the transformation of $A$ into $S$ derived from $T$. Such a transformation

Presented to the Society, September 3, 1947; received by the editors June 10, 1947.

${ }_{1}^{1}$ Numbers in brackets refer to the references cited at the end of the paper. 
has often been considered in the study of fixed point properties of continua as for instance by Alexandroff and Hopf [1, p. 479]. If $T$ is a continuous transformation of a subset $A$ of $E$ into a subset $B$ of $E, A$ will be said to have property ( $\left.\mathrm{b}^{\prime}\right)$ with respect to $T$ if $T$ leaves no point of $A$ invariant and the transformation $T^{\prime}$ of $A$ into $S$ derived from $T$ is homotopic to a constant mapping.

LEMma 1. There does not exist a bounded plane continuum $M$ which has property $\left(\mathrm{b}^{\prime}\right)$ with respect to a continuous interior transformation $T$ of $M$ onto a topological 2-cell I which contains $M$.

Proof. Suppose the lemma is not true and that there does exist such a bounded plane continuum $M$. Without loss of generality we may take $I$ to be the circular 2-cell in the Cartesian plane with center at $o$, radius 1 , and boundary $S$. Let $T^{\prime}$ designate the transformation of $M$ into $S$ derived from $T$. By assumption, $M$ has property $\left(\mathrm{b}^{\prime}\right)$ with respect to $T$ and hence $T^{\prime}$ is homotopic to a constant mapping. By a theorem of Eilenberg [2, p. 168, Theorem 1'] there exists a continuous real valued function $\Phi$ which transforms $M$ into a subset of $R$, the set of real numbers, such that for each point $x$ of $M, T^{\prime}(x)$ is the point $e^{i \Phi(x)}$. Since $M$ is closed and bounded, $\Phi(M)$ is a bounded and closed subset of $R$. For each point $x$ of $I$, let $X$ designate the set consisting of all real numbers $\Phi(y)$, for $y$ belonging to $T^{-1}(x)$, and let $\theta(x)$ be the greatest lower bound of the set $X$. Since $T$ is an interior continuous transformation, it follows by a lemma of Eilenberg [2, p. 174] that $T^{-1}$ is a continuous multi-valued transformation of $I$ onto $M$, and hence that $\theta$ is a continuous transformation of $I$ into $R$. For each point $x$ of $I$ let $x z$ designate the directed half line with initial point at $x$ which is parallel in direction and opposite in sense to the directed line segment $o y$, where $y$ is the point $e^{i \theta(x)}$, and let $T_{2}(x)$ be the first point on $x z$ distinct from $x$ which $x z$ has in common with $S$. We know $T_{2}(x)$ exists for each point $x$ in $I$ since $M$ is a subset of $I$ and since, from the continuity of $\theta$ and $T^{-1}$ and the assumption that $T$ leaves no point of $M$ invariant, it follows that there is a point of $M$ distinct from $x$ lying on $x z$ and hence a point of $S$ distinct from $x$ lying on $x z$. Thus, since $\theta$ is a continuous transformation of $I$ into $R$, it follows that $T_{2}$ is a continuous transformation of $I$ into the boundary of $I$ which leaves no point of $I$ invariant. But this contradicts the Brouwer fixed point theorem for 2-cells. The lemma is therefore true.

THEOREM 1. If $T$ is an interior continuous transformation of a locally connected unicoherent bounded plane continuum $M$ onto a topological 2-cell which contains $M$, then $T$ leaves a point of $M$ invariant. 
Proof. For suppose $T$ leaves no point of $M$ invariant. Then the continuous transformation $T^{\prime}$ of $M$ into $S$ derived from $T$ exists and, by a theorem of Eilenberg [2, p. 168, Theorem $\left.6^{\prime}\right]$, since $M$ is unicoherent and locally connected, $M$ has property (b), and hence has property $\left(b^{\prime}\right)$ with respect to $T$. But this contradicts Lemma 1 .

CoROllaRy. If $T$ is an interior continuous transformation of a bounded locally connected plane continuum which does not separate the plane onto a topological 2-cell which contains $M$, then $T$ leaves a point of $M$ invariant.

Proof. For a plane continuum which does not separate the plane is unicoherent.

THEOREM 2. If $T$ is an interior continuous transformation of a topological 2-cell $I$ onto a continuum $M$ which contains $I$, then $T$ leaves a point of I invariant.

Proof. Suppose $T$ leaves no point of $I$ invariant. Then the derived transformation $T^{\prime}$ exists and, since $I$ is unicoherent, it follows by the previously cited theorem of Eilenberg that $I$ has property (b) and hence has property $\left(\mathrm{b}^{\prime}\right)$ with respect to $T$. But $T$ transforms some subset $N$ of $I$ onto $I$. Then $N$ has property ( $\left.\mathrm{b}^{\prime}\right)$ with respect to $T$. But this contradicts Lemma 1.

It is easy to construct an example to show that Theorem 2 would not be true if the condition that $T$ be interior were omitted from the hypothesis of the theorem.

Applications of the theorems of this paper to the theory of functions of a complex variable are suggested by the fact that a function of a complex variable, analytic in a region $R$, defines an interior continuous transformation of $R$ into a subset of the complex plane.

\section{REFERENCES}

1. P. Alexandroff and H. Hopf, Topologie, Berlin, 1935.

2. S. Eilenberg, Sur les transformations d'espace métrique en circonférence, Fund. Math. vol. 24 (1935) pp. 160-176.

Orlahoma Agricultural and Mechanical College 